\title{
El arpa, agua que cae: metáfora y simbolismo sonoro como estrategias de formación de palabras en el léxico de instrumentos musicales en guaraní
}

Estefanía Baranger

Universidad de Buenos Aires - Consejo Nacional de Investigaciones Científicas y Técnicas, Buenos Aires, Argentina

estefaniabaranger@gmail.com

Recepción: marzo 2020. Aceptación: junio 2020.

\section{Resumen}

El campo semántico de los instrumentos musicales, a diferencia de la nomenclatura etnobotánica y etnozoológica, representa un área aún poco explorada por los estudios etnolingüísticos. El presente trabajo parte del análisis de tres lexemas primarios; mbaraka, angu'a y mimby, que originalmente en mbya guaraní designaban instrumentos musicales de origen indígena y, posteriormente, en guaraní antiguo y guaraní moderno, o bien fueron re-semantizados o incluidos en compuestos para designar instrumentos introducidos por la población de origen europeo. Cada fuente consultada, entonces, representa un distinto grado de contacto con la cultura envolvente, factor que se refleja en las relaciones de hiperonimia e hiponimia entre los lexemas primarios y las incorporaciones o desarrollos más recientes. El análisis lingüístico asimismo revela que las estrategias de formación de palabras en este campo resultan en nombres morfológicamente complejos que encuentran su motivación en relaciones de base analógica o metafórica con otros elementos del mundo, así como instancias de simbolismo sonoro, fenómenos usualmente asociados a la nomenclatura biológica.

Palabras clave: guaraní, etnomusicología, léxico, contacto lingüístico, etnolingüística 


\title{
A harpa, água que cai: metáfora e simbolismo sonoro como estratégias de formação de palavras no léxico de instrumentos musicais em Guarani
}

\begin{abstract}
Resumo
O campo semântico dos instrumentos musicais, diferentemente da nomenclatura etnobotânica e etnozoológica, representa uma área ainda pouco explorada pelos estudos etnolinguísticos. 0 presente trabalho baseia-se na análise de três lexemas primários; mbaraka, angu'a e mimby, que originalmente no Guarani Mbya designavam instrumentos musicais de origem indígena e, posteriormente, no Guarani Antigo e Guarani Moderno, foram ressemantizados ou incluídos em palavras compostas para designar instrumentos introduzidos pela população de origem europeia. Cada fonte consultada, portanto, representa um grau diferente de contato com a cultura circundante, fator que se reflete nas relações de hiperonímia e hiponímia entre os lexemas primários e suas mais recentes incorporações ou desenvolvimentos. A análise linguística também revela que as estratégias de formação de palavras nesse campo resultam em nomes morfologicamente complexos que encontram a sua motivação em relações de base analógica ou metafórica com outros elementos presentes no mundo, assim como instâncias de simbolismo sonoro, fenômenos geralmente associados à nomenclatura biológica.
\end{abstract}

Palavras-chave: Guarani, etnomusicologia, léxico, contato linguístico, etnolinguística.

\section{The Harp, a Waterfall: Metaphor and Sound Symbolism as Word-formation Strategies in the Musical Instrument Lexicon in Guarani}

\begin{abstract}
The semantic field of musical instruments, unlike ethnobotanical and ethnozoological nomenclature, still represents an underexplored area in ethnolinguistic studies. The present work is based on the analysis of three primary lexemes; mbaraka, angu'a and mimby, which originally in Mbya Guarani designated musical instruments of indigenous origin and, later, in Old Guarani and Modern Guarani, were either re-semantisized or included in nominal compounds in order to designate instruments introduced by the population of European origin. Each source, then, represents a different degree of contact with the creole culture, a fact that is reflected in
\end{abstract}


the hyponymy and hypernymy relations between these primary lexemes and the more recent incorporations or developments. Furthermore, this linguistic analysis reveals word-formation strategies in this field result in morphologically complex names motivated by metaphorical or analogical relations with other elements of the world, as well as instances of sound symbolism, phenomena which are usually associated with biological nomenclature.

Keywords: Guarani, ethnomusicology, lexicon, linguistic contact, ethnolinguistics

La vibrante alveolar [r],y la lateral líquida [l],íntimamente relacionadas desde el punto de vista fonético, parecen ser inconscientemente los sonidos más apropiados del inventario del habla humana para servir de segmentos en nombres de anuros (tanto ranas como sapos), de manera que los nombres de estas criaturas se forman así en una proporción extraordinaria de lenguas. ${ }^{1}$

(Berlin, 1992)

Es como si la habilidad de comprender nuestra experiencia por medio de la metáfora fuese un sentido más, como la vista, el tacto, o el oído, con la metáfora como único medio para percibir y experimentar gran parte del mundo. La metáfora es tan parte de nuestro funcionamiento como el sentido del tacto, y tan precioso también. ${ }^{2}$

(Lakoff y Johnson, 1980)

\section{Introducción}

La enigmática naturaleza del vínculo entre las cosas y sus nombres, necesariamente arbitraria en los signos de Ferdinand de Saussure o motivada en los íconos de Charles Peirce, constituye una problemática tal vez más

\footnotetext{
${ }^{1}$ The alveolar thrill [r], and the phonetically closely related lateral liquid [l], seem to be unconsciously drawn upon as the most appropriate sounds from the human speech inventory to serve as segments for the names of anurans (both frogs and toads), so that the names of these creatures are formed in this fashion in an extraordinary proportion of languages. (Berlin, 1992, p. 250).

${ }^{2}$ It is as though the ability to comprehend experience through metaphor were a sense, like seeing or touching or hearing, with metaphors providing the only ways to perceive and experience much of the world. Metaphor is as much a part of our functioning as our sense of touch, and as precious. (Lakoff y Johnson, 1980, p. 486).
} 
filosófica que lingüística, cuyos antecedentes se remontan al Crátilo de Platón y aún perdura en la actualidad. Los estudios sobre simbolismo sonoro, en este sentido, recuperan la existencia de una relación motivada entre sonido y significado. Así, en -R- is for FROG, Brent Berlin (1992) analiza un corpus de casi treinta lenguas en el que se evidencia una fuerte tendencia translingüística a codificar los nombres de ranas y sapos con el fonema [r], y, análogamente, Ohala (1997) identifica una correlación entre vocales cerradas [i] y nombres de animales de tamaño pequeño, y vocales abiertas [a] para seres de mayor tamaño. Estos nombres, entonces, no constituyen lexemas enteramente arbitrarios. En la misma línea, en Metaphors we live by (1980), Lakoff y Johnson, cuyas palabras también sirven de epígrafe al presente trabajo, explican cómo la metáfora no constituye un mero recurso estético limitado al lenguaje poético, sino que forma parte del lenguaje cotidiano y, al igual que la analogía, cuenta con una alta capacidad explicativa. En este sentido, ambas figuras resultan herramientas sumamente útiles en la creación de ítems léxicos para nombrar nuevos referentes en relación con otros pre-existentes, con los que los primeros guardan algún tipo de nexo a nivel cognitivo. Así, la existencia de nombres con base analógica o metafórica en las lenguas naturales también nos aleja del terreno de la más absoluta arbitrariedad en relación al dilema de las cosas y sus nombres.

El presente trabajo aborda el campo semántico de los instrumentos musicales en tres lenguas ${ }^{3}$ genética y geográficamente relacionadas, y está basado íntegramente en fuentes secundarias: mbya-guaraní (Cadogan,

\footnotetext{
${ }^{3}$ Según la primera clasificación propuesta por Aryon D. Rodrigues (1958), el mbya, el guaraní antiguo y el guaraní moderno se incluyen genealógicamente dentro del Grupo I de la familia tupí, rama tupí-guaraní. Si bien el etnónimo mbya que las comunidades emplean para autodesignarse comienza a ser utilizado en la literatura en el siglo XX, los mbya actuales pueden ser fácilmente identificados como los cainguás de las crónicas jesuíticas -y de los posteriores escritos de Ambrosetti (1895)-, denominados así precisamente debido a su rechazo al contacto y la imposibilidad de evangelizarlos (Cebolla Badie, 2016). Y, en efecto, la literatura especializada concuerda en que el mbya, dentro de la familia tupi-guaraní, es de las lenguas históricamente menos afectadas por el contacto lingüístico ya que, al contrario de lo que sucedió con otras etnias guaraníes locales, los jesuitas de la Compañía de Jesús no lograron atraerlos a las misiones (Cadogan, 1968 y Dietrich, 1993). Paralelamente, durante el siglo XVI en el área ocupada por las misiones jesuíticas se podían identificar dos dialectos del guaraní claramente diferenciados; el guaraní antiguo, empleado en las reducciones, documentado y normalizado por los jesuitas, y el guaraní hablado por los mestizos -hijos de colonos españoles y madres guaraníes- y españoles, que se consagró como lengua general empleada en ámbitos urbanos fuera de las misiones (Rodrigues, 1996). Las diferencias entre ambas lenguas, si bien numerosas, se debían principalmente a los cambios en el guaraní urbano por causa del contacto con el español (Gómez Rendón, 2008). Rodrigues (1996), asimismo, establece la filiación genética directa entre esta lengua general y el guaraní criollo o moderno hablado actualmente en Paraguay. En la actualidad, mientras que el guaraní antiguo es considerado una lengua extinta, el guaraní moderno goza del estatus de lengua oficial en Paraguay desde el año 1992, y el mbya es hablado principalmente por población indígena tanto en Argentina como en Brasil y Paraguay.
} 
2011 [1992]), guaraní antiguo (Montoya, 2011 [1639] y Restivo, 1893 [1722]) y guaraní moderno (Canese y Alcaraz, 1997 y Sanabria, 2007). Nuestro objetivo es describir el cambio léxico-semántico que experimentaron las palabras que integran esta área específica del vocabulario y los mecanismos disponibles para crear nuevas palabras, tanto desde un nivel morfo-fonológico, como desde las motivaciones que los atraviesan. Además nos proponemos destacar la relevancia del estudio de esta porción de vocabulario, raramente abordada en las investigaciones etnolingüísticas, con base en que todas las sociedades poseen prácticas musicales que conforman su identidad cultural y se encuentran materializadas en instrumentos musicales nativos cuya nomenclatura constituye una porción de vocabulario altamente resistente a préstamos de otras lenguas.

El escrito está organizado en tres secciones. La primera se centra en el análisis del cambio léxico-semántico que sufrieron tres lexemas primarios centrales: mbaraka, mimby y angu'a, cuyos referentes y extensión semántica varían en cada período, reflejando distintos grados de contacto con la sociedad criolla o europea. La segunda sección aborda las estrategias involucradas en la creación de nuevos términos en este campo semántico, y cómo las relaciones de hiponimia e hiperonimia entre los lexemas primarios y los compuestos resultantes de base analógica o metafórica re-organizan las taxonomías nativas. En la tercera sección se analizan instancias de simbolismo sonoro, fenómeno que al igual que los compuestos ya mencionados, nos obliga a pensar en la existencia de otro tipo de motivaciones en la relación entre las palabras que integran este campo semántico y los instrumentos que designan.

\section{Cambio léxico-semántico: ampliación y restricción de significados}

\subsection{Mbaraka: de sonajera a guitarra}

En mbya (Cadogan, 2011 [1992]) el término mbaraka denota esencialmente instrumentos idiófonos, es decir, distintos tipos de sonajeras -incluso el cascabel de las serpientes-, a excepción del compuesto mbaraka pytã, para referir a la guitarra:

\begin{tabular}{cc}
\hline mbaraka & 'sonajera' \\
\hline mbaraka mirĩ & 'sonajera ritual' \\
sonajera pequeño & \\
\hline $\begin{array}{c}\text { mbaraka pytã } \\
\text { sonajera rojo }\end{array}$ & 'guitarra común' \\
\hline
\end{tabular}




\begin{tabular}{cc}
\hline $\begin{array}{c}\text { mbói } \text { mbaraka-kue } \\
\text { víbora sonajera-PST }\end{array}$ & 'cascabel del crotalo' \\
\hline $\begin{array}{c}\text { mbaraka ju } \\
\text { sonajera amarillo }\end{array}$ & 'sonajera áurea' \\
\hline
\end{tabular}

En guaraní antiguo, por otro lado, si bien se conserva la definición original de mbaraka como instrumento idiófono, se destaca que la extensión semántica del lexema abarcaría ya en este período "todo instrumento músico" (Montoya, 2011, p. 328). Los ejemplos citados por Montoya, sin embargo, remiten casi exclusivamente a instrumentos de cuerda:

[...] mbaraka sã cuerdas [de instrumentos], mbaraka sã moatyrõ templar, ojoja katu mbaraka están templados los instrumentos [...], mbaraka sã osog quebrose la cuerda, amombipyu mbaraka sã/amoyvyrakuãndog mbaraka sã destemplar o aflojar la cuerda, mbaraka sã pokaháva/moatãháva clavijas, mbaraka sã menda puente de la guitarra, mbaraka'y ku'akuaháva trastes, ajapypy mbaraka sã poner los dedos en las cuerdas, añatõi mbaraka sã tocar rasgado, aikyty mbaraka sã tocar rabeles con arco, mbaraka revi sã la cuerda de que penden todas las cuerdas de los rabeles, guyrapa mbaraka sã kytykáva el arco de rabeles (2011, p. 329).

El sentido idiófono está directamente ausente en Restivo (1893 [1722]), donde mbaraca se encuentra en la entrada de "guitarra", junto a préstamos del español como vihuela y discante, y encontramos también compuestos específicos que denotan otros instrumentos de cuerda como mbaracaguaçu para arpa; y mbaraca ycuágui minîbae para violín o rabel. Por otro lado, en las fuentes de guaraní moderno se incorporan aún más hispanismos para referir a instrumentos de cuerda; en (Canese y Alcaraz, 1997) ravel y arapa, y en Sanabria (2007) rrave, e incluso el compuesto rraverusu para referir al violonchelo. En relación al lexema que nos compete, en la definición de (Canese y Alcaraz, 1997) conviven ambos sentidos "guitarra, matraca, maraca" en la misma entrada, pero en Sanabria (2007) finalmente desaparece esta ambigüedad, $y$, mediante al desplazamiento de la sílaba tónica, se distinguen dos palabras claramente identificables: mbaráka, para denotar el instrumento idiófono, y mbaraka, para la guitarra, con acentuación paroxítona y oxítona, respectivamente.

\footnotetext{
${ }^{4}$ Las glosas no constan en las fuentes originales, son de mi autoría. Las abreviaturas empleadas siguen las convenciones establecidas por el Max Planck Institute for Evolutionary Anthropology (Leipzig): 3 tercera persona; IN paradigma inactivo; DIM diminutivo; SUB subordinante; NMLZ nominalización; PST pasado; LOC locativo.
} 
En el Handbook of South American Indians (1948), Alfred Métraux ya da cuenta de que las guitarras españolas están progresivamente suplantando a los instrumentos nativos guaraníes, así como Ambrosetti (1895) en su etnografía de los cainguás documenta la construcción de violines y guitarras por los indígenas, imitando los instrumentos europeos. El exhaustivo trabajo de investigación de Irma Ruiz (1996) resulta clave en este sentido; la investigadora traza una distinción entre la guitarra española de seis cuerdas y la guitarra mbya, claramente de inspiración española, pero de cinco cuerdas y con otra afinación. Este instrumento, empleado en contextos religiosos -a diferencia de la guitarra criolla con la que sólo se interpretaban músicas paraguayas de moda-, se ejecuta de un modo específico privilegiando el factor rítmico al melódico o armónico, lo que funcionalmente la acerca más a los idiófonos que a los cordófonos, como lo describe Ruiz:

[...] ésta es sostenida con la mano y el brazo izquierdos en posición vertical, o aproximadamente así. Ello es posible debido a que la única técnica utilizada en las expresiones musicales a cargo de los mismos es el rasgueo con las cuerdas al aire. Teniendo en cuenta que éste se realiza en forma continua, el movimiento se asemeja al del sacudir de una maraca (1996, p. 87).

Asimismo, el desplazamiento semántico que sufre el lexema mbaraka, desde su sentido originario idiófono en mbya, hasta emplearse actualmente para referir a instrumentos de cuerda y principalmente a la guitarra en guaraní moderno, podría deberse también a su naturaleza privilegiada de "instrumento que sirve para acompañar el canto", que es resaltada en las definiciones de Montoya (2011 [1639]) y Sanabria (2007). Podemos, así, pensar en la guitarra española como el equivalente cultural de la maraca acompañando el canto en la cultura guaraní, de ahí que en un primer lugar se extienda a "todo instrumento músico" (Montoya 2011 [1639]) y vuelva a restringirse para referir exclusivamente a guitarra en las fuentes modernas, ante el surgimiento de neologismos o la incorporación de hispanismos para cubrir los otros elementos del sistema.

\subsection{Mimby: de maderas a metales}

En relación a los aerófonos, en Cadogan (2011 [1992]) mimby refiere exclusivamente a flautas, tanto el lexema primario como los compuestos registrados por el autor, constituyendo estos últimos distintos tipos de flautas. En Montoya (2011 [1639]), por otro lado, se extiende el significado de mimby para abarcar "flauta, chirimía, fagot", y en la misma fuente encontramos los compuestos mimby tarara/ mimby terere, para referir a 
"trompeta, clarín". Es decir, se plantea una distinción entre instrumentos de viento madera e instrumentos de viento metal, característica de la orquestación occidental dependiendo del material empleado tradicionalmente para su construcción, claramente ausente en el sistema indígena donde bajo este rótulo solo se contemplan las flautas tradicionales hechas de tacuara (Ambrosetti, 1895). En relación a las fuentes posteriores a Montoya, en Restivo (1893 [1722]) el significado de mimby y mimby tarara se restringe otra vez a "flauta" y "trompeta", respectivamente, y el compuesto mimby terere, al parecer, cae en desuso, ya que en fuentes posteriores directamente no se registra. Actualmente, según las fuentes de guaraní moderno, mimby tarara aún refiere exclusivamente a "trompeta", empero mimby abarcaría otros instrumentos viento madera como "pito" (Canese y Alcaraz, 1997) y “quena” (Sanabria, 2007).

\subsection{Angu'a: de mortero a tambor}

En cuanto a lo que a los membranófonos refiere, el lexema angu'a en la mayoría de las fuentes refiere tanto a "tambor" como a "mortero" (Cadogan, 2011 [1992], Montoya, 2011 [1639], Canese y Alcaraz, 1997) o exclusivamente a "mortero" (Sanabria, 2007 y Restivo, 1893 [1722]). La relación entre los términos podría deberse a que ambos se componen de dos elementos; un recipiente hueco de madera de tamaño grande -en el caso del tambor con una membrana que es percutida-, y cuentan con un mazo para ejecutar el acto de moler o percutir. Dado que es la primera o única acepción explicitada en las fuentes consultadas, podemos asumir que "mortero" fue en efecto el significado original y posteriormente se produjo la extensión semántica a "tambor" por analogía, dadas las semejanzas a nivel morfológico entre ambos instrumentos y su forma de ejecución. Inclusive en Sanabria (2007) se explicita la distinción entre angu'a como "mortero" y el neologismo angu'a-pu (literalmente: "sonido de mortero") para "bombo, tambor" y angu'a-pu-rusu para instrumentos más grandes del mismo tipo.

\section{Estrategias de formación de palabras}

El campo de los instrumentos musicales presenta mecanismos de formación de palabras que involucran los niveles morfosintáctico y fonológico, y que son frecuentes en otros campos del léxico. A continuación se presentan la diversas estrategias formales mediante a las cuales los hablantes construyen nuevas palabras que designan instrumentos originalmente ausentes en el léxico nativo. Estas involucran lexemas pre-existentes cuya combinación resulta en compuestos de base analógica o metafórica, en el primer caso basados en similitudes con otros instrumentos musicales 
a nivel tamaño, forma o modo de ejecución, y en el segundo en las sensaciones que evocan los sonidos que estos producen.

a) Nombre + modificador. Estrategia sumamente productiva, en especial en mbya, donde encontramos, por ejemplo, el lexema primario mimby que funciona como categoría genérica "flauta", dentro de la cual pueden especificarse elementos menores del sistema mediante al empleo del diminutivo $i$ o la yuxtaposición de otro elemento que actúa como modificador del nombre principal indicando una propiedad, como es el caso de puku o guachu donde los compuestos resultantes designan distintos tipos de flauta indicando diferencias de tamaño o forma:

\begin{tabular}{cc}
\hline lexema primario & $\mathrm{N}+$ modificador \\
\hline & mimby puku \\
mimby & flauta largo \\
"flauta" & flauta larga" \\
& mimby guachu \\
(mbya) & flauta grande \\
& "flauta de hombre" \\
\cline { 2 - 2 } & mimby $\boldsymbol{i}$ \\
& flauta DIM \\
& flauta de mujer" \\
\hline
\end{tabular}

El caso de mimby en mbya constituye un caso prototípico en que el lexema primario y el hiperónimo coinciden, mientras que los compuestos se encuentran en relación de hiponimia. Análogo es el caso de angu'a-purusu en guaraní moderno, donde el compuesto designa meramente un tipo de bombo (angu'a-pu) más grande. El modificador rusu también es empleando en guaraní moderno (Sanabria, 2007) modificando el lexema rrave, no para referir a un subtipo de violín sino para designar otro instrumento de cuerdas, el violonchelo, así como guasu/guachu figura en fuentes del guaraní antiguo (Restivo, 1893 [1722]) y moderno (Canese y Alcaraz, 1997) modificando el lexema primario mbaraka, para referir al arpa. En ambos casos se evidencia que los elementos modificados, si bien no se encuentran en relación de hiponimia con "violín" y "guitarra", fueron de incorporación más tardía al vocabulario guaraní.

\begin{tabular}{cc}
\hline lexema primario & $\mathrm{N}+$ modificador \\
\hline $\begin{array}{c}\text { 'guaraca } \\
\text { (guaraniantiguo) }\end{array}$ & $\begin{array}{l}\text { mbaraca-guaçu } \\
\text { guitarra-grande } \\
\text { 'arpa' }\end{array}$ \\
\hline $\begin{array}{c}\text { rrave } \\
\text { 'violín' }\end{array}$ & $\begin{array}{c}\text { rrave-rusu } \\
\text { violín-grande } \\
\text { 'violonchelo' }\end{array}$ \\
\hline
\end{tabular}


En estos últimos casos, sin embargo los compuestos guardan efectivamente alguna relación con el lexema primario como el modo de generar sonido -pulsando cuerdas o mediante a un arco- o semejanzas a nivel morfológico, empero también encontramos casos donde el lexema primario y el nombre modificado designan instrumentos completamente distintos como mbaraka y mbaraka-pytã en mbya (Cadogan, 2011 [1992]), sonajera y guitarra respectivamente, como ya fue especificado en la sección anterior.

b) Cláusulas reducidas. En las fuentes consultadas encontramos solo dos instancias de esta estrategia, en ambos casos para referir al violín en relación a la guitarra, evidentemente de incorporación más temprana:

\author{
Guarani moderno \\ (Canese y Alcaraz, 1997) \\ mbaraca-'i h-asẽ-ngy \\ guitarra-DIM 3.IN-llorar-SUB \\ 'violín'
}

\author{
Guarani antiguo \\ (Restivo, 1893 [1722]) \\ mbaraca y-cuá-gui minî-bae \\ guitarra 3.IN-pozo-LOC \\ pequeño-NMLZ \\ 'violín/rabel'
}

Así, el violín se define como una "guitarra pequeña que llora", o una "guitarra de cavidad pequeña”. En el primer caso mediante a una personificación del instrumento musical, al que se le adjudican la acción de llorar, en el segundo se lo define en oposición a la guitarra, instrumento de cavidad mayor. El empleo de cláusulas nominalizadas con base metafórica ya fue identificado como un recurso altamente productivo para formar fitónimos en otras lenguas de la familia tupi-guarani (ver González, 2011, para tapiete), así como en otras lenguas amerindias (ver Navarro Hartmann, 2016, para mapuche).

c) Verbo no finito: Los compuestos que involucran la yuxtaposición de una raíz verbal sin marcación personal y el lexema $p u$ "sonido" para generar nombres de instrumentos musicales constituyen una estrategia algo marginal; en las fuentes consultadas los casos conviven con sinónimos de uso evidentemente más difundido y sólo encontramos una instancia de registro de cada uno.

\author{
Guarani moderno \\ (Canese y Alcaraz, 1997) \\ guata-pu \\ caminar-sonido \\ "tambor, trompeta"
}

\author{
Guarani moderno \\ (Sanabria, 2007) \\ mbota-pu \\ golpear-sonido \\ "tambor"
}


Guarani moderno (Canese y Alcaraz, 1997)

$y$-sa-pu

agua-chorro-sonido

"arpa"

Sanabria (2007) registra el compuesto mbotapu para "tambor", donde el verbo no finito "golpear" refiere a la manera en que el sonido es producido por el ejecutante. Canese y Alcaraz (1997), por su parte, documentan el compuesto guatapu, que referiría tanto al tambor, como la trompeta, significados que raramente se agrupan bajo un mismo rótulo. La extensión del verbo "caminar" para referir a la sección rítmica se apoya en una metáfora de base corporal, pensando las figuras rítmicas como si fueran pasos, en la misma línea que opera el walking del contrabajo en el jazz. Sin embargo, en este caso también podría indicar que esos instrumentos pueden ser tocados caminando, ya que, en efecto, una de las características que guardan en común los instrumentos de viento metal y percusión es que son los que componen en esencia las bandas de música de los desfiles militares, lo que podría haber motivado estos compuestos en un primer lugar. Por otro lado, en el compuesto ysapu (arpa), que puede traducirse literalmente como "sonido de chorro de agua", la metáfora que prima se basa en la percepción humana del sonido producido por el instrumento designado que evoca el ruido de gotas o chorros de agua cayendo.

d) Adaptación fonológica de préstamos del español. Esta constituye una estrategia altamente productiva en situaciones que implican contacto lingüístico, y consiste en la incorporación al vocabulario de la lengua receptora de términos de la lengua mayoritaria que designan objetos inexistentes al momento de contacto. En las fuentes consultadas encontramos préstamos del español como vihuela y discante para referir a la guitarra en guaraní antiguo (Montoya, 2011 [1639]) que no sufrieron modificaciones a nivel fonológico, probablemente porque solo convivieron con su sinónimo mbaraca durante un primer período y cayeron en desuso sin incorporarse al vocabulario de la lengua receptora, dado que no figuran en las fuentes modernas. Sin embargo, en las fuentes consultadas también encontramos casos de préstamos re-fonologizados del español, es decir, adaptados fonológicamente según el inventario de sonidos disponibles del guaraní; tal es el caso de arapa para referir al "arpa” en guaraní moderno (Canese y Alcaraz, 1997), donde se inserta una vocal epentética /a/, que en este caso coincide con la vocal de la sílaba anterior, para adaptar el hispanismo a la estructura silábica guaraní que no admite secuencias de consonantes ni sílabas trabadas. El caso de ravel en guaraní moderno (Canese y Alcaraz, 1997) también resulta interesante en este aspecto; donde la fricativa 
bilabial /b/ del original en español "rabel" se vuelve labiodental /v/, y, a su vez, el mismo préstamo recibe posteriormente otra modificación ortográfica en rrave (Sanabria, 2007), donde el grafema "rr" es empleado para codificar la vibrante múltiple, ausente en el inventario fonológico guaraní, pero obligatoria en el contexto de inicio de sílaba en español.

\section{Simbolismo sonoro imitativo y convencionalizado: el caso de mimby tarara}

En el compuesto ampliamente extendido para referir a 'trompeta', encontramos las formas tarara y terere (Montoya, 2011 [1639]), que también ameritan sendas propias entradas en el vocabulario de Montoya. Claramente de origen onomatopéyico, los términos guardan iconicidad con un sonido originalmente no lingüístico y son incluidos en el inventario léxico de la lengua para referir o evocar diversos eventos sonoros; en el caso de tarara "quebrantar el maíz en el mortero; hacer frangollo" (Montoya, 2011 [1639], p. 530) y “dar diente contra diente" (Restivo, 1893 [1722], p. 215), o en el caso de terere "crujir, ruido de lo que se quiebra" (Montoya, 2011 [1639], p 565; Restivo, 1893 [1722], p. 483). Estos últimos casos se englobarían en lo que se denomina simbolismo sonoro imitativo, dada la relación icónica -empero translingüísticamente impredecible (Beck, 2008, p. 5)- entre forma fonológica y significado. No obstante, en el caso del compuesto para referir a instrumentos de viento propongo analizar estas formas como casos de simbolismo sonoro convencionalizado, dado que, si bien no se hace evidente una relación entre las formas aisladas tarará/tereré y los compuestos de los que participan, la relación entre sonido y significado tampoco parece ser arbitraria en este caso. Como ya fue referido en la introducción, existen tendencias en las lenguas del mundo a codificar ciertos significados con elementos fonológicos específicos, como ilustra la siguiente síntesis de Ohala:

\begin{tabular}{cc}
\hline Formas fonológicas & Significado \\
\hline - vocales altas posteriores & " consonantes sordas \\
- tonos altos (en lenguas tonales) & "pequeño" \\
\hline - vocales bajas anteriores & \\
- consonantes sonoras & "grande" \\
- tonos bajos (en lenguas tonales) & \\
\hline
\end{tabular}

Esquema en base a Ohala (1997).

Retomando esta propuesta, podemos analizar las vocales abiertas /a/ y /e/ en mimby tarara/terere como indicadores de una flauta de mayor tamaño. No obstante, teniendo en cuenta el carácter altamente composicional de 
la lengua, esta hipótesis tampoco parece convincente dada la facilidad con la que los hablantes nativos o criollos pueden crear el compuesto mimby-guachu para este fin, ya presente en mbya como "flauta de hombre" (Cadogan, 2011 [1992]). Sin embargo, el factor tamaño sí puede resultar relevante si tenemos en cuenta que, así como la diferencia de tamaño entre las cajas de resonancia de los instrumentos de cuerda y los tractos vocales masculinos y femeninos resultan en diferencias a nivel registro, en los instrumentos de viento una mayor tubería equivale a un registro más grave y viceversa. La trompeta de por sí tiene un registro más grave que la flauta, $y$, aunque la distancia entre ambos no parezca tan significativa, lo cierto es que el registro preferido de cada instrumento es muy diferente; dentro de su registro la trompeta presenta más homogeneidad en los medios -las notas más agudas presentan restricciones en cuanto a dinámicas-, y la flauta, al contrario va ganando definición y brillo a medida que asciende a las notas más agudas.

Así, podemos plantear que la onomatopeya aporta la plantilla o template consonántico, conformado por los fonemas / $\mathrm{t} / \mathrm{y} / \mathrm{r} /$, que se rellena con las vocales abiertas /a/ y /e/ siguiendo las tendencias del simbolismo sonoro convencionalizado, no en relación al tamaño del instrumento sino al registro del mismo. Las vocales abiertas en /tVrVrV/ representarían entonces sonidos graves, y las mimby tarara podrían interpretarse como flautas que producen sonidos en un registro más bajo que sus predecesoras, las mimby a secas. Asimismo, la asociación entre tonos graves y vocales abiertas, y tonos agudos y vocales cerradas, también es explorada por Ohala (1997) que los agrupa en lenguas tonales para codificar significados "grande" y "pequeño", respectivamente. No resulta entonces desquiciado plantear que en las lenguas no tonales la distinción entre sonidos graves y agudos mantenga vínculos estrechos con las mismas formas fonológicas. Abonan a esta hipótesis también: (i) el empleo de tarara y terere como variantes, que nos aleja del simbolismo sonoro imitativo u onomatopéyico en el cual la vocal que represente más fielmente el sonido real sería elegida por sobre la otra; (ii) la existencia de turututu en guaraní moderno (Sanabria, 2007) para referir al clarín, un instrumento de registro más agudo que el de la trompeta, cuyo sonido, por ende, se codifica mediante una vocal alta /u/; y (iii) el empleo de tarara en el compuesto angu'a tarara (Canese y Alcaraz, 1997) para referir al bombo, en oposición a agu'a para a tambores y timbales, dado que, mientras que los timbales se incluyen en el grupo de instrumentos membranófonos que tienen una afinación determinada aguda, el bombo y el tambor no tienen una afinación determinada pero sí un timbre grave y agudo, respectivamente. Así, 
siguiendo la hipótesis propuesta, no parece casual que el instrumento que designa el compuesto con tarara sea el membranófono más grave de la sección rítmica.

\section{Observaciones finales}

El presente trabajo se enfocó en el campo semántico de los instrumentos musicales en tres lenguas genéticamente relacionadas de la familia tupiguarani. La preferencia por un enfoque diacrónico radica en entender el léxico de una lengua como un sistema abierto y adaptativo, capaz de actualizarse ante la incorporación o el desarrollo de nuevos elementos. En este sentido, resulta interesante destacar la relativa escasez préstamos del español en nuestro corpus y la preferencia por el desarrollo de nuevos términos a partir del reciclaje de lexemas primarios que son re-interpretados o re-combinados para producir nuevas palabras. Asimismo, en el corpus analizado puede observarse que tanto el cambio léxico-semántico que sufrieron los lexemas primarios, como el desarrollo de compuestos de base analógica o metafórica, conllevan una re-organización de las taxonomías tradicionales a nivel sistema para incluir elementos de naturaleza ajena. En segundo lugar, el análisis propuesto evidenció que en este campo operan los mismos procesos morfo-fonológicos de formación de palabras que en otras áreas del léxico, a pesar de que éste no constituya una porción de vocabulario usual en los estudios etnolingüísticos, como sí lo son el léxico etnobotánico o etnozoológico (González, 2011, Cúneo, 2010 y Navarro Hartmann, 2016). Se ha demostrado que los nombres morfológicamente complejos en este campo semántico constituyen formas motivadas que expresan una relación de base analógica o metafórica con otros elementos del mundo. Si bien este recurso se presenta también en otros campos semánticos, las metáforas específicas que asocian los sonidos producidos por los instrumentos con otros eventos sensoriales (como el ruido del agua en ysapu, el sonido de los pasos en guatapu, o el llanto en mbaraca'i hasẽngy) sí son especificas de este campo y, en esta línea, sería interesante profundizar en la extensión de estas asociaciones cognitivas desde una perspectiva translingüística. Finalmente, el caso de mimby tarara ilustra otro tipo de motivación en la formación de unidades lexicales, y cómo la identificación de patrones translingüísticamente regulares para codificar determinados significados permite explorar la lógica que subyace a nombres a simple vista etimológicamente opacos. 


\section{Bibliografía}

》Ambrosetti, J. B. (1895). Los indios cainguá del Alto Paraná (Misiones). Boletín del Instituto Geográfico Argentino,15, 661-744.

» Beck, D. (2008). Ideophones, Adverbs, and Predicate Qualification in Upper Nexaca Totonac. International Journal of American Linguistics 74(1), 1-46.

" Berlin, B. (1992). Ethnobiological Classification: Principles of Categorization of Plants and Animals in Traditional Societies. New Jersey: Princeton University Press.

»Cadogan, L. (1968). Chono Kybwyra: aporte al conocimiento de la mitología guaraní. Suplemento Antropológico de la Revista del Ateneo Paraguayo, 3, 55158.

» Cadogan, L. (2011 [1992]). Diccionario Mbyá-Guaraní/Castellano. Asunción: CEPAG/CEADUC.

»Canese, N. K de, y Alcaraz, F. A. (1997). Ñe'ẽryru. Asunción: Instituto Superior de Lenguas, Universidad Nacional de Asunción.

»Cebolla Badie, M. (2016). Cosmología y naturaleza mbya-guaraní. Buenos Aires: Biblos.

»Cúneo, P. (2010). Nombres que "suenan": simbolismo sonoro en el léxico etnobiológico toba. En C. Messineo, G. F. Scarpa y F. Tola (Comps.). Léxico y categorización etnobiológica en grupos indígenas del Gran Chaco (pp. 65-81). Santa Rosa: Universidad Nacional de La Pampa.

»Dietrich, W. (1993). Mbya, guarani criollo y castellano: El contacto de las tres lenguas en un grupo mbyá de Misiones. Signo \& Seña, 3, 56-71.

» Gómez Rendón, J. (2008). Typological and Social Constraints on Language Contact: Amerindian Languages in Contact with Spanish (tesis de doctorado en Lingüística Teórica). Netherlands Graduate School of Linguistics, Amsterdam, Holanda.

" González, H. A. (2011). Léxico etnobotánico tapiete (tupí-guaraní), lengua del Chaco argentino. INDIANA, 28, 255-288.

» Lakoff, G. y Johnson, M. (1980). Metaphors We Live by. Chicago: University of Chicago Press.

" Métraux, A. (1948). The Guarani. En J. H. Steward (Ed.). Handbook of South American Indians, vol. 3 (pp. 69-94). Washington: Bureau of American Ethnology.

» Montoya, A. R. de (2011 [1639]). Tesoro de la lengua guaraní [Edición de F. Grünberg y B. Meliá]. Asunción: CEPAG.

» Navarro Hartmann, H. (2016). Arbitrariedad y motivación en el léxico etnobiológico del mapuche (tesis de doctorado en Filosofía y Letras). Universidad Nacional de La Plata, Facultad de Humanidades y Ciencias de la 
Educación, La Plata, Argentina.

"Ohala, J. (1997). Sound Symbolism. Proc. 4th Seoul International Conference on Linguistics [SICOL], 11-15 Aug 1997, 98-103.

» Restivo, P. (1893 [1722]). Vocabulario de la lengua guaraní [Edición de C. F. Seybold].

» Rodrigues, A. D. (1958). Classification of Tupi-Guarani. International Journal of American Linguistics, 24, 231-234.

»Rodrigues, A. D. (1996). As línguas gerais sul-americanas. PAPIA, 4 (2), 6-18. Recuperado de http://revistas.fflch.usp.br/papia/article/view/1791/1602

» Ruiz, I. (1996). Acerca de la sustitución de un idiófono indígena por un cordófono europeo: los mbaraká de los mbyá-guaraní. Revista argentina de musicología, 1, 81-92.

"Sanabria, L. T. (2007). Mi pequeño avañe'ê de mochila. Diccionario bilingüe guaraní-castellano/castellano-guaraní. Asunción: Editorial Occidente.

\section{Biografía/Biografia/Biography}

\section{Estefanía Baranger}

Música. Licenciada en Letras por la Universidad de Buenos Aires, donde actualmente cursa el Doctorado en Lingüística, y becaria del Consejo Nacional de Investigaciones Científicas y Técnicas. Realizó parte de su formación en Brasil, tanto durante el grado (UNICAMP) como posgrado (UFRJ). Su área es la antropología lingüística y, actualmente, su investigación se centra en la descripción gramatical de la lengua mbya-guaraní hablada en la provincia de Misiones (Argentina), donde hace trabajo de campo desde el año 2017. Sus intereses también incluyen las lenguas en contacto, la etnomusicología, la etnobiología y la lutería de instrumentos de cuerda. 